\title{
MULTIPOINT FOCAL BOUNDARY VALUE PROBLEMS ON INFINITE INTERVALS ${ }^{1}$
}

\author{
S. UMAMAHESWARAM and M. VENKATA RAMA ${ }^{2}$ \\ University of Hyderabad \\ School of Mathematics and Computer Information Sciences \\ Central University P.O. \\ Hyderabad-500 194, INDIA
}

\begin{abstract}
For the differential equation $y^{(n)}=f(x, y)$, we state a set of necessary and sufficient conditions for the existence of a solution $(i)$ on a semi-infinite interval for a $k$-point right focal boundary value problem and $(i i)$ on $(-\infty, \infty)$ for a $(n-1)$-point right focal boundary value problem. The conditions are in terms of the existence of a pair of solutions $u(x), v(x)$ satisfying some auxiliary boundary conditions and algebraic inequatilities.
\end{abstract}

Key words: Focal boundary value problems.

AMS (MOS) subject classifications: $\quad 34 \mathrm{~B} 15$.

\section{INTRODUCTION}

Let $n$ be a fixed positive integer greater than $1, k, n(1), \ldots, n(k)$ be arbitrary but fixed integers satisfying $1<k \leq n, n(1) \geq 2,1 \leq n(r) \leq n-1, r=2, \ldots, k, n(1)+\ldots+n(k)=n$, and $x_{1}<\ldots<x_{k}$ be arbitrary real numbers. Define $s(0)=0$ and $s(r)=n(1)+\ldots+n(r)$ for $r=1, \ldots, k$.

In this paper we obtain in Theorems 2.3 and 3.1 necessary and sufficient conditions for the existence of a solution $(i)$ on the interval $\left(-\infty, x_{1}\right]$ of the $k$-point right focal boundary value problem $(B V P)(1.1),(1.2)$ with $(r, i) \neq(1,0)$ and $(i i)$ on the interval $(-\infty, \infty)$ of the $B V P$ (1.1), (1.3) with $i \neq m$, the underlying equations being

$$
\begin{gathered}
y^{(n)}=f(x, y) \\
y^{(i)}\left(x_{r}\right)=y_{r i}, r=1, \ldots, k \\
i=s(r-1), \ldots, s(r)-1
\end{gathered}
$$

\footnotetext{
${ }^{1}$ Received: October, 1991. Revised: December, 1991.
}

${ }^{2}$ This author is supported by a Post-Doctoral Research Associateship of the University Grants Commission, New Delhi, India. 
and

$$
y^{(i)}\left(x_{1}\right)=y_{i}, i=0, \ldots, n-1
$$

These conditions are stated in terms of the existence of a pair of solutions $u(x), v(x)$ of (1.1) satisfying some auxiliary boundary conditions $(B C s)$ and algebraic inequalities. We assume throughout this paper that the differential equation (1.1) satisfies some of the following hypotheses.

A. $\quad f$ is continuous on $\mathbb{R}^{2}$.

$U R$. Solutions of $n$-point right focal $B V P \mathrm{~s}$, if they exist, are unique; that is, if $y(x), z(x)$ are solutions of the $B V P(1.1),(1.2)$ with $x_{1}<\ldots<x_{k}$ and $k=n$ then $y(x) \equiv z(x)$ on $\left[x_{1}, x_{k}\right]$.

$U$. Solutions of initial value problems (IVPs) are unique.

E. $\quad$ All solutions of (1.1) exist on $(-\infty, \infty)$.

$E^{\prime}$. All solutions of (1.1) exist on $(-\infty, c)$, where $-\infty<c \leq \infty$ is a constant depending on the solution.

Some existence theorems on infinite intervals for conjugate $B V P$ s have been proved for the cases $n=2$ and 3 in [5,6] and for arbitrary $n$ in [7]. However, existence theorems on infinite intervals for focal $B V P$ s do not seem to be included in the literature so far.

\section{AN EXISTENCE THEOREM FOR SEMI-INFINITE INTERVALS}

We first prove the following lemma which is useful in the proofs of our main theorems.

Lemma 2.1: Assume the hypotheses $A, U R$, and $E^{\prime}$ hold. Let $\ell, m$ be arbitrary but fixed integers with $1 \leq \ell \leq k, s(\ell-1) \leq m \leq s(\ell)$, and $(\ell, m) \neq(1,0)$. Suppose $u(x)$, $v(x)$ are distinct solutions of the $B V P(1.1),(1.2)$ with $(r, i) \neq(1,0),(\ell, m)$ and satisfying $u\left(x_{0}\right)=v\left(x_{0}\right)$ for some $x_{0}<x_{1}$ and $w(x) \equiv u(x)-v(x)$. Then

$$
\begin{aligned}
& w^{\prime}(x) \neq 0 \text { for } x_{0} \leq x<x_{1}, w(x) \neq 0 \text { for } x_{0}<x \leq x_{1} . \\
& w^{(s(r-1))}(x) \neq 0 \text { for } \quad x_{r-1} \leq x<x_{r}, \quad w^{(s(r-1)-1)}(x) \neq 0 \quad \text { for } x_{r-1}<x \leq x_{r}, \\
& r=2, \ldots, \ell-1 \text {. } \\
& w^{(s(\ell-1))}(x) \neq 0 \text { for } x_{\ell-1} \leq x<x_{\ell}, w^{(m)}(x) \neq 0 \text { for } x_{\ell-1}<x \leq x_{\ell} .
\end{aligned}
$$

Proof: If $w^{\prime}\left(x^{\prime}\right)=0$ for some $x^{\prime}, x_{0} \leq x^{\prime} \leq x_{1}$ then using the $B C$ s (1.2), successive applications of Rolle's theorem to $w^{\prime}, \ldots, w^{(m-1)}$ on appropriate subintervals of $\left[x^{\prime}, x_{\ell}\right]$ and the theorem in [3] result in the contradiction $w \equiv 0$. Thus the first inequality in (i) holds.

Now $w^{\prime}(x) \neq 0$ for $x_{0}<x<x_{1}$ implies $w(x) \neq 0$ for $x_{0}<x \leq x_{1}$. 
The proofs for (ii) and (iii) are similar.

We also need the following lemma due to Kolmogorov [4] which is stated here for the sake of convenience.

Lemma 2.2: Let $M>0,[a, b] \subset \mathbb{R}$ and $y(x) \in C^{n}[a, b]$ be an arbitrary function with the property that $|y(x)| \leq M$ and $\left|y^{(n)}(x)\right| \leq M$ on $[a, b]$. Then there exists a constant $K>0$ depending on $M$ and the interval $[a, b]$ such that $\left|y^{(r)}(x)\right| \leq K$ on $[a, b]$ for $1 \leq r \leq n-1$.

Theorem 2.3: Assume the hypotheses $A, U R, U$ and $E^{\prime}$ hold. Let $\ell, m$ be arbitrary but fixed integers with $1 \leq \ell \leq k, s(\ell-1) \leq m \leq s(\ell)-1$ and $(\ell, m) \neq(1,0)$. Then a necessary and sufficient condition that the BVP (1.1), (1.2) with $(r, i) \neq(1,0)$ has a solution $y(x)$ on $\left(-\infty, x_{k}\right]$ is that there exist solutions $u(x), v(x)$ of $(1.1)$ on $\left(-\infty, x_{k}\right]$ satisfying the condition (1.2) with $(r, i) \neq(1,0),(\ell, m)$;

$$
u(x) \geq v(x) \text { on }\left(-\infty, x_{1}\right]
$$

and

$$
(-1)^{m} u^{(m)}\left(x_{\ell}\right) \leq(-1)^{m} y_{\ell_{m}} \leq(-1)^{m} v^{(m)}\left(x_{\ell}\right) .
$$

In the sufficiency part the solution $y(x)$ satisfies $u(x) \geq y(x) \geq v(x)$ on $\left(-\infty, x_{1}\right]$.

Proof:

Necessity: This is obvious since we can choose $u(x)=v(x)=y(x)$ where $y(x)$ is the assumed solution of $(1.1),(1.2)$ with $(r, i) \neq(1,0)$.

Sufficiency: If $(-1)^{m} y_{\ell_{m}}=(-1)^{m} u^{(m)}\left(x_{\ell}\right) \quad$ (or $\left.(-1)^{m} v^{(m)}\left(x_{\ell}\right)\right)$ we can choose $y(x)=u(x)($ or $v(x))$ and there is nothing to prove. Suppose

$$
(-1)^{m} u^{(m)}\left(x_{\ell}\right)<(-1)^{m} y_{\ell m}<(-1)^{m} v^{(m)}\left(x_{\ell}\right) .
$$

If $u\left(x^{\prime}\right)=v\left(x^{\prime}\right)$ for some $x^{\prime}<x_{1}$, then since $u(x) \geq v(x)$ on $\left(-\infty, x_{1}\right]$ we must have $u^{\prime}\left(x^{\prime}\right)=v^{\prime}\left(x^{\prime}\right)$ and this contradicts Lemma $2.1(i)$. Hence $u(x)>v(x)$ on $\left(-\infty, x_{1}\right)$. For $j=1,2, \ldots$, let $v_{j}(x)$ be the solution of the $B V P(1.1),(1.2)$ with $(r, i) \neq(1,0)$ and $y\left(x_{1}-j\right)=v\left(x_{1}-j\right)$ which exists by theorem 3 of [2].

Now we claim that $v_{j}^{\prime}\left(x_{1}-j\right)>v^{\prime}\left(x_{1}-j\right)$. Clearly $v_{j}^{\prime}\left(x_{1}-j\right) \neq v^{\prime}\left(x_{1}-j\right)$ by Lemma 2.1 (i). Also, due to the same reason, if $v_{j}^{\prime}\left(x_{1}-j\right)<v^{\prime}\left(x_{1}-j\right)$ then $v_{j}^{\prime}(x)<v^{\prime}(x)$ for all $x$, $x_{1}-j<x<x_{1}$. Let $g(x) \equiv v_{j}(x)-v(x)$ so that $g^{\prime}(x)<0$ on $\left[x_{1}-j, x_{1}\right), g^{(i)}\left(x_{1}\right)=0$, $i=1, \ldots, s(1)-1$ and by Lemma $2.1(i i), g^{(s(1))}\left(x_{1}\right) \neq 0$. Hence for $x_{1}-j \leq x<x_{1}$ we have by Taylor's theorem 


$$
\begin{gathered}
-1=\operatorname{Sgn} g^{\prime}(x) \\
=\operatorname{Sgn}\left(g^{\prime}(x)-g^{\prime}\left(x_{1}\right)\right) \\
=\operatorname{Sgn}\left\{\frac{\left(x-x_{1}\right)^{s(1)-1}}{(s(1)-1) !} g^{(s(1))}\left(x_{1}\right)\right\} .
\end{gathered}
$$

This implies Sgn $g\left({ }^{(s(1))}\left(x_{1}\right)=(-1)^{s(1)}\right.$ and by Lemma $2.1(i i), S g n g^{(s(1))}(x)=(-1)^{s(1)}$ for $x_{1}<x<x_{2}$. Further $g^{(i)}\left(x_{2}\right)=0$ for $i=s(1), \ldots, s(2)-1$ and by Lemma $2.1(i i) g^{(s(2))}\left(x_{2}\right) \neq 0$. Hence for $x_{1}<x<x_{2}$, we again by Taylor's theorem

$$
\begin{aligned}
& (-1)^{s(1)}=\operatorname{Sgn} g^{(s(1))}(x) \\
= & \operatorname{Sgn}\left(g^{(s(1))}(x)-g^{(s(1))}\left(x_{2}\right)\right) \\
= & \operatorname{Sgn}\left\{\frac{\left(x-x_{2}\right)^{s(2)-s(1)}}{(s(2)-s(1)) !} g^{(s(2))}\left(x_{2}\right)\right\} .
\end{aligned}
$$

Thus $\operatorname{Sgn} g^{(s(2))}\left(x_{2}\right)=(-1)^{s(2)}$ and consequently by Lemma $2.1(i i) \operatorname{Sgn} g^{(s(2))}(x)=(-1)^{s(2)}$ for $x_{2}<x<x_{3}$. Continuing this argument through the intervals $\left[x_{2}, x_{3}\right], \ldots,\left[x_{\ell-1}, x_{\ell}\right]$ we obtain $S g n \quad g^{(s(r))}\left(x_{r}\right)=(-1)^{s(r)}, \quad r=1, \ldots, \ell-1 \quad$ and, by Lemma $2.1 \quad$ (iii), Sgn $g^{(s(\ell-1))}(x)=(-1)^{s(\ell-1)}, x_{\ell-1}<x<x_{\ell}$ whereas $g^{(i)}\left(x_{\ell}\right)=0, \quad i=s(\ell-1), \ldots, m-1$. Again an application of Taylor's theorem yields that for $x_{\ell-1}<x<x_{\ell}$

$$
\begin{aligned}
& (-1)^{s(\ell-1)}=S g n g^{(s(\ell-1))}(x) \\
= & S g n\left(g^{(s(\ell-1))}(x)-g^{(s(\ell-1))}\left(x_{\ell}\right)\right) \\
= & S g n \frac{\left(x-x_{\ell}\right)^{m-s(\ell-1)}}{(m-s(\ell-1)) !} g^{(m)}\left(x_{\ell}\right) \\
= & (-1)^{m-s(\ell-1)} \operatorname{Sgn} g^{(m)}\left(x_{\ell}\right) .
\end{aligned}
$$

Thus $\operatorname{Sgn} g^{(m)}\left(x_{\ell}\right)=(-1)^{m}$ or $(-1)^{m}\left(v_{j}-v\right)^{(m)}\left(x_{\ell}\right)>0$, a contradiction to the inequality (2.1). Hence the claim $v_{j}^{\prime}\left(x_{1}-j\right)>v^{\prime}\left(x_{1}-j\right)$ is true. This implies by Lemma $2.1(i)$ $v_{j}(x)>v(x)$ on $\left[x_{1}-j, x_{1}\right]$ for all $j$.

Next we claim that $v_{j}(x)<u(x)$ for $x_{1}-j \leq x \leq x_{1}$. If $v_{j}\left(x^{\prime}\right)=u\left(x^{\prime}\right)$ holds for some $x^{\prime}$, $x_{1}-j<x^{\prime}<x_{1}$ then $v_{j}^{\prime}\left(x^{\prime}\right) \geq u^{\prime}\left(x^{\prime}\right)$. However $v_{j}^{\prime}\left(x^{\prime}\right) \neq u^{\prime}\left(x^{\prime}\right)$ by Lemma $2.1(i)$. On the other hand, if $v_{j}^{\prime}\left(x^{\prime}\right)>u^{\prime}\left(x^{\prime}\right)$ holds for some $x^{\prime}, x_{1}-j<x^{\prime}<x_{1}$, then by Lemma 2.1 (i) we should have $v_{j}^{\prime}(x)>u^{\prime}(x)$ for $x^{\prime} \leq x \leq x_{1}$. However if $h(x) \equiv v_{j}(x)-u(x), x^{\prime} \leq x \leq x_{1}$ then $h^{\prime}(x)>0$ 
for $x^{\prime} \leq x<x_{1}, h^{(i)}\left(x_{1}\right)=0, i=1, \ldots, s(1)-1$ and $h^{(s(1))}\left(x_{1}\right) \neq 0$. Hence for $x^{\prime}<x<x_{1}$, we have by Taylor's theorem

$$
\begin{gathered}
1=\operatorname{Sgn} h^{\prime}(x) \\
=\operatorname{Sgn}\left(h^{\prime}(x)-h^{\prime}\left(x_{1}\right)\right) \\
=(-1)^{s(1)-1} \operatorname{Sgn} h^{(s(1))}\left(x_{1}\right) .
\end{gathered}
$$

Thus, $\operatorname{Sgn} h^{(s(1))}\left(x_{1}\right)=(-1)^{s(1)-1}$. Continuing the arguments as in the case of $\left(v_{j}-v\right)$ in the earlier part of the proof we obtain, for $x_{\ell-1}<x<x_{\ell}$,

$$
\begin{gathered}
\operatorname{Sgn}\left(v_{j}-u\right)^{(m)}\left(x_{\ell}\right)=S g n h^{(m)}\left(x_{\ell}\right) \\
=(-1)^{m-1} .
\end{gathered}
$$

Thus, $(-1)^{m-1} v_{j}^{(m)}\left(x_{\ell}\right)>(-1)^{m-1} u^{(m)}\left(x_{\ell}\right)$, a contradiction to the inequality $(2.1)$ and hence the claim is true.

Furthermore, since $v_{j}(x)$ are solutions of equation (1.1), it follows by hypothesis $U R$ and the theorem in [3] that for each $j=1,2, \ldots, v(x)<v_{j}(x)<v_{j+1}(x)<u(x)$ on $\left[x_{1}-j, x_{1}\right.$ ]. By Lemma 2.2 and Kamke's convergence theorem [p.14, 1] there exists a subsequence called again $\left\{v_{j}(x)\right\}$ and a solution $v_{0}(x)$ of $(1.1)$ such that $v_{j}^{(i)}(x) \rightarrow v_{0}^{(i)}(x), i=0, \ldots, j-1$ uniformly on compact subintervals of $\left(-\infty, x_{1}\right]$. Now the solution $y(x)=v_{0}(x)$ has the desired properties.

\section{AN EXISTENCE THEOREM FOR $(-\infty, \infty)$}

In this section we assume the additional hypothesis $U L$,

$U L: \quad$ Solutions of $n$-point left focal $B V P \mathrm{~s}$, if they exist, are unique; that is, if $y(x), z(x)$ are solutions of the $B V P(1.1),(1.2)$ with $x_{k}<\ldots<x_{1}$ and $k=n$ then $y(x) \equiv z(x)$ on $\left[x_{k}, x_{1}\right]$.

Theorem 3.1: Assume the hypotheses $A, U R, U L, U$ and $E$ hold. Let $m$ be a fixed but arbitrary integer with $1 \leq m \leq n-1$. Then a necessary and sufficient condition for the $B V P$ (1.1), (1.3) with $i \neq 0$ to have a solution $y(x)$ on $(-\infty, \infty)$ is that there exist solutions $u(x)$, $v(x)$ of $(1.1)$ on $(-\infty, \infty)$ satisfying the conditions (1.3) with $i \neq 0, m$,

$$
u(x) \geq v(x) \text { on }(-\infty, \infty)
$$

and

$$
(-1)^{m} u^{(m)}\left(x_{1}\right) \leq(-1)^{m} y_{m} \leq(-1)^{m} v^{(m)}\left(x_{1}\right)
$$


In the sufficiency part, the solution $y(x)$ satisfies $u(x) \geq y(x) \geq v(x)$ on $(-\infty, \infty)$.

\section{Proof:}

Necessity: This is obvious since we can choose $u(x)=v(x)=y(x)$.

Sufficiency: If $(-1)^{m} y_{m}=(-1)^{m} u^{(m)}\left(x_{1}\right)$ (or $(-1)^{m} v^{(m)}\left(x_{1}\right)$ ) we can choose $y(x)=u(x)$ (or $v(x))$ and there is nothing to prove.

Suppose the inequality

$$
(-1)^{m} u^{(m)}\left(x_{1}\right)<(-1)^{m} y_{m}<(-1)^{m} v^{(m)}\left(x_{1}\right)
$$

holds. Then we have $u(x)>v(x)$ on $\left(-\infty, x_{1}\right)$ as in Theorem 2.1. Furthermore, if $u\left(x^{\prime}\right)=v\left(x^{\prime}\right)$ for some $x^{\prime}>x_{1}$ we can arrive at a contradiction by virtue of the hypothesis $U L$ and a lemma analogous to Lemma 2.1 for left focal $B C$ s. Hence $u(x)>v(x)$ holds for all $x \neq x_{1}$.

If for each $j \geq 1, v_{j}(x)$ is the solution of the $B V P(1.1),(1.3)$ with $i \neq 0$ and $y\left(x_{1}-j\right)=v\left(x_{1}-j\right)$ then as in Theorem 2.1 , we have $v(x)<v_{j}(x)<v_{j+1}(x)<u(x)$ on $\left[x_{1}-j, x_{1}\right]$. Similarly, for each $j \geq 1$ we can obtain a solution $u_{j}(x)$ of $(1.1),(1.3)$ with $i \neq 0$ and $y\left(x_{1}-j\right)=u\left(x_{1}-j\right)$ with the property that $u_{j+1}(x)<u_{j}(x) \leq u(x)$ on $x_{1}-j \leq x \leq x_{1}$. Moreover, by the hypothesis $U R$ and the theorem of [3] it follows that for each $j, v_{j}(x)<u_{j}(x)$ on $\left[x_{1}-j, x_{1}\right)$. Thus we have for each $j \geq 1, v(x)<v_{j}(x)<v_{j+1}(x)<u_{j+1}(x)<u_{j}(x)<u(x)$ on $\left[x_{1}-j, x_{1}\right)$. Now since $u_{j}(x), v_{j}(x)$ are solutions of equation (1.1) it follows by Lemma 2.1 and Kamke's convergence theorem [p. 14, 1] that there exists subsequences of $\left\{u_{j}(x)\right\},\left\{v_{j}(x)\right\}$ called again $\left\{u_{j}(x)\right\},\left\{v_{j}(x)\right\}$ such that $u_{j}(x) \rightarrow u_{0}(x), v_{j}(x) \rightarrow v_{0}(x)$ uniformly on compact subintervals of $\left(-\infty, x_{1}\right)$; consequently $u_{0}(x), v_{0}(x)$ are solutions of the $B V P(1.1)$, (1.3) with $i \neq 0$ satisfying $v(x) \leq v_{0}(x) \leq u_{0}(x) \leq u(x)$ on $\left(-\infty, x_{1}\right.$ ]. Similarly, using the hypothesis $U L$, the results analogous to the theorem in [3], theorem 3 of [2], and Lemma 2.1 for left focal $B V P \mathrm{~s}$, we can obtain a pair of solutions $w_{0}(x), z_{0}(x)$ of (1.1), (1.3) with $i \neq 0$ satisfying $v(x) \leq w_{0}(x) \leq z_{0}(x) \leq u(x)$ on $\left[x_{1}, \infty\right)$

Now the four quantities $u_{0}\left(x_{1}\right), v_{0}\left(x_{1}\right), w_{0}\left(x_{1}\right)$ and $z_{0}\left(x_{1}\right)$ can be ordered in one of the following ways,

$$
\begin{aligned}
& v_{0}\left(x_{1}\right) \leq u_{0}\left(x_{1}\right) \leq w_{0}\left(x_{1}\right) \leq z_{0}\left(x_{1}\right) \\
& w_{0}\left(x_{1}\right) \leq z_{0}\left(x_{1}\right) \leq v_{0}\left(x_{1}\right) \leq u_{0}\left(x_{1}\right) \\
& w_{0}\left(x_{1}\right) \leq v_{0}\left(x_{1}\right) \leq u_{0}\left(x_{1}\right) \leq z_{0}\left(x_{1}\right) \\
& v_{0}\left(x_{1}\right) \leq w_{0}\left(x_{1}\right) \leq z_{0}\left(x_{1}\right) \leq u_{0}\left(x_{1}\right) .
\end{aligned}
$$

In any case let $y(x)$ be the solution of the IVP (1.1), (1.3) with $y\left(x_{1}\right)=c_{0}$, where $c_{0}$ is the 
average of the middle two quantities in the appropriate ordering stated above. They $y(x)$ is the desired solution.

Remark 1: It follows from theorem 3 of [2] and the theorem in [3] that Theorems 2.3 and 3.1 will be true if we replace (1.1) by

$$
y^{(n)}=f\left(x, y, \ldots, y^{(n-1)}\right)
$$

provided the hypothesis $A$ is replaced by the hypothesis $A^{\prime}$ and the additional hypothesis $C$ (compactness of solutions of $\left.(1.1)^{\prime}\right)$ holds where $A^{\prime}$ and $C$ are as follows:

$A^{\prime}$ : $\quad f$ is continuous on $\mathbb{R}^{n+1}$.

$C: \quad$ If $\left\{y_{k}(x)\right\}$ is a sequence of solutions of $(1.1)$ and $[c, d]$ is a compact subinterval of $(a, b)$ such that $\left\{y_{k}(x)\right\}$ is uniformly bounded on $[c, d]$, then there exists a subsequence $\left\{y_{k_{j}}(x)\right\}$ such that $\left\{y_{k_{j}}^{(i)}(x)\right\}$ converges uniformly on $[c, d], 0 \leq i \leq n-1$.

Remark 2: In the case $n=3$, the hypothesis $C$ can be omitted in view of the comments on page 990 of [2]; while in the case $n=2$, the hypotheses $U$ and $C$ can be omitted in view of theorem 3.1 of [8].

\section{REFERENCES}

[1] P. Hartman, “Ordinary Differential Equations”, Wiley, New York (1964).

[2] J. Henderson, "Existence of solutions of right focal point boundary value problems for ordinary differential equations", J. of Nonlinear Analysis: Theory, Methods and Appl. 5, (1981), pp. 989-1002.

[3] _ _ Uniqueness of solutions of right focal point boundary value problems for ordinary differential equations, J. of Differential Equations 41, (1981), pp. 218-227.

[4] A. Kolmogorov, "On inequalities between the upper bounds of the successive derivatives of an arbitrary function on an infinite interval, Amer. Math. Soc. Transl. Ser. 14, (1949), pp. 3-19.

[5] K. Schrader, "Existence theorems for second order boundary value problems", J. of Differential Equations 5, (1969), pp. 572-584.

[6] _- "Second and third order boundary value problems", Proc. of Amer. Math. Soc. 32, (1972), pp. 247-252.

[7] S. Umamaheswaram, "Boundary value problems for higher order differential equations", J. of Differential Equations 18, (1975), pp. 188-201.

[8] S. Umamaheswaram and M. Venkata Rama, "Focal subfunctions and second order differential inequalities", Rocky Mountain Journal of Math. (to appear). 


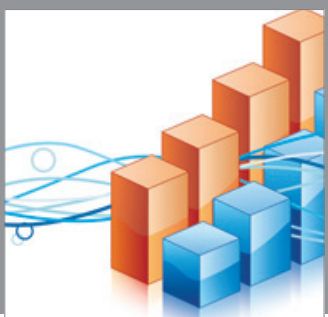

Advances in

Operations Research

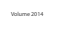

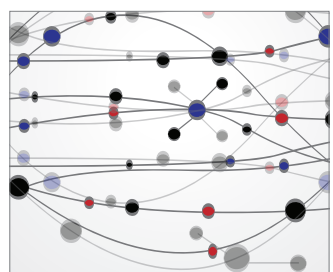

\section{The Scientific} World Journal
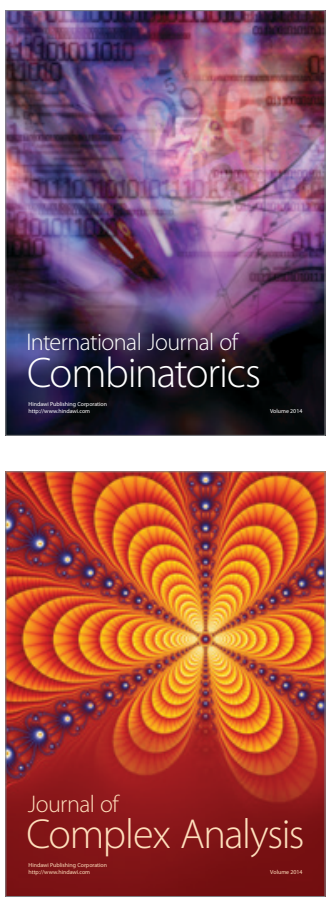

International Journal of

Mathematics and

Mathematical

Sciences
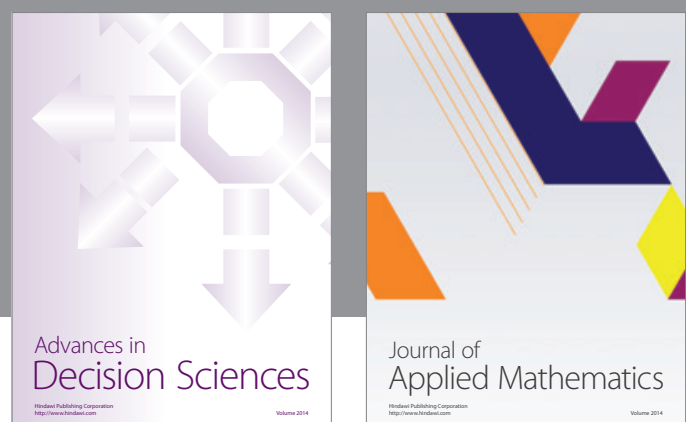

Journal of

Applied Mathematics
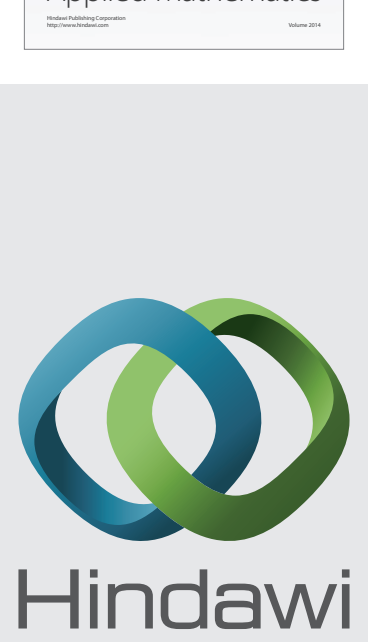

Submit your manuscripts at http://www.hindawi.com
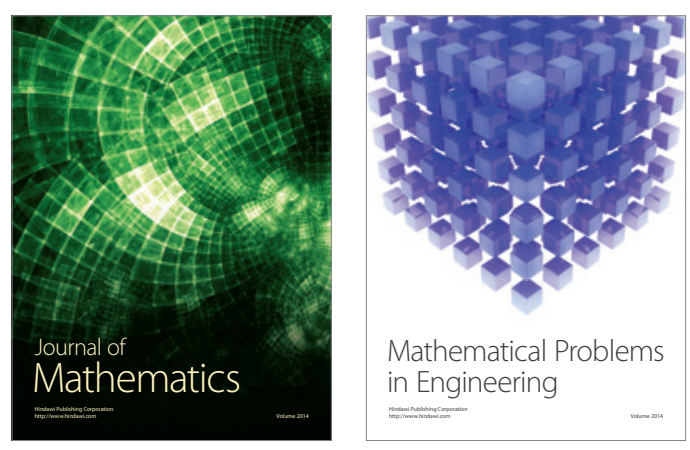

Mathematical Problems in Engineering
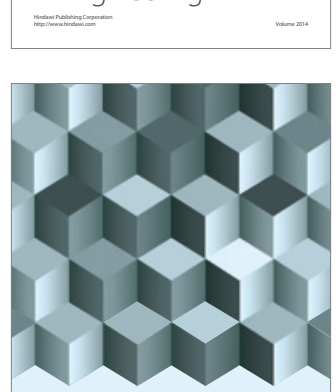

Journal of

Function Spaces
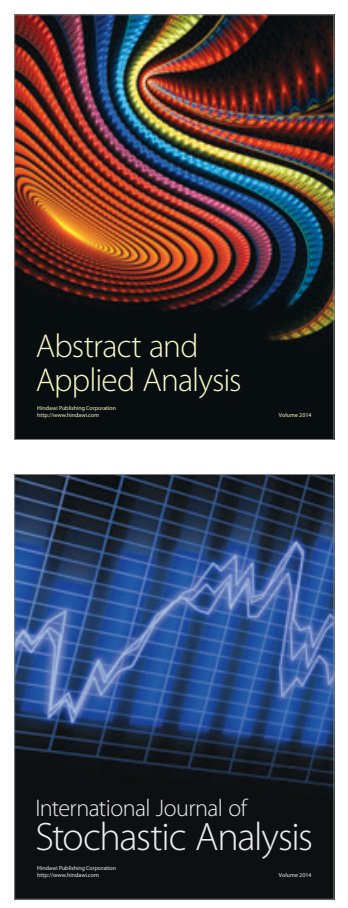

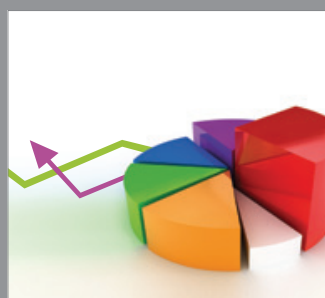

ournal of

Probability and Statistics

Promensencen
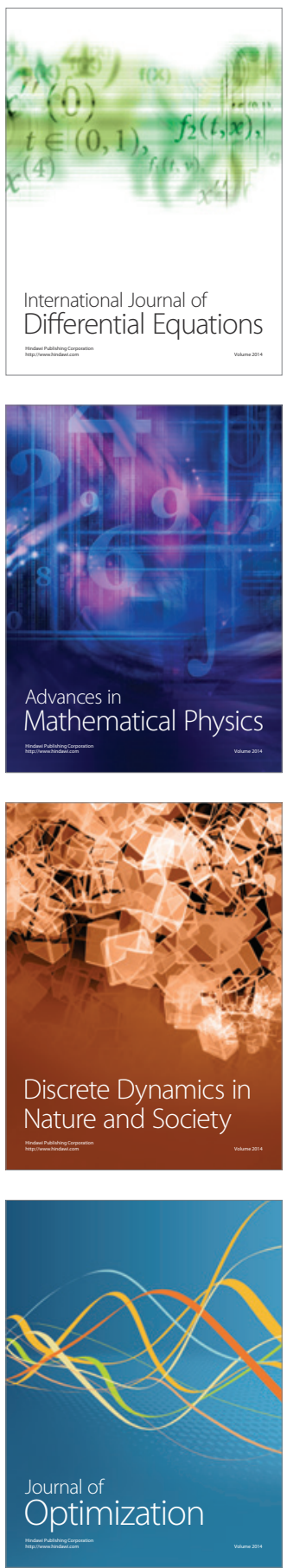